\title{
Poly(ADP-ribosylation) is present in murine sciatic nerve fibers and is altered in a Charcot-Marie-Tooth-1E neurodegenerative model
}

\author{
Laura I Lafon Hughes ${ }^{\text {Corresp., }}{ }^{1}$ ， Carlos J Romeo Cardeilliac ${ }^{2}$ ， Karina B Cal Castillo ${ }^{2}$, Salomé C Vilchez Larrea ${ }^{3}$ \\ José R Sotelo Sosa ${ }^{2}$ ， Gustavo A Folle Ungo ${ }^{1}$ ， Silvia H Fernández Villamil ${ }^{3,4}$ ， Alejandra E Kun González ${ }^{\text {Corresp. } 2,5}$ \\ 1 Departamento de Genética, Instituto de Investigaciones Biológicas Clemente Estable (IIBCE), Montevideo, Uruguay \\ 3 \\ 3 Instituto de Investigaciones en Ingeniería Genética y Biología Molecular “Dr. Héctor N. Torres, Consejo Nacional de Investigaciones Científicas y \\ Técnicas, Buenos Aires, Argentina \\ 4 Departamento de Química Biológica, Facultad de Farmacia y Bioquímica, Universidad de Buenos Aires, Buenos Aires, Argentina \\ 5 Departamento de Biología Celular y Molecular, Sección Bioquímica, Facultad de Ciencias, Universidad de la República, Montevideo, Uruguay \\ Corresponding Authors: Laura I Lafon Hughes, Alejandra E Kun González \\ Email address: lauralafon2010@gmail.com, akun@fcien.edu.uy
}

Background. Poly-ADP-ribose (PAR) is a polymer synthesized by poly-ADP-ribose polymerases (PARPs) as a postranslational protein modification and catabolized mainly by poly-ADP-ribose glycohydrolase (PARG). In spite of the existence of cytoplasmic PARPs and PARG, research has been focused on nuclear PARPs and PAR, demonstrating roles in the maintenance of chromatin architecture and the participation in DNA damage responses and transcriptional regulation. We have recently detected non-nuclear PAR structurally and functionally associated to the E-cadherin rich zonula adherens and the actin cytoskeleton of VERO epithelial cells. Myelinating Schwann cells (SC) are stabilized by E-cadherin rich autotypic adherens junctions (AJ). We wondered whether PAR would map to these regions. Besides, we have demonstrated an altered microfilament pattern in peripheral nerves of Trembler-J (Tr-J) model of CMT1-E. We hypothesized that cytoplasmic PAR would accompany such modified F-actin pattern.

Methods. Wild-type (WT) and Tr-J mice sciatic nerves cryosections were subjected to immunohistofluorescence with anti-PAR antibodies (including antibody validation), F-actin detection with a phalloidin probe and DAPI/DNA counterstaining. Confocal image stacks were subjected to a colocalization highlighter and to semi-quantitative image analysis.

Results. We have shown for the first time the presence of PAR in sciatic nerves. Cytoplasmic PAR colocalized with F-actin at non-compact myelin regions in WT nerves. Moreover, in Tr-J, cytoplasmic PAR was augmented in close correlation with actin. In addition, nuclear PAR was detected in WT SC and was moderately increased in Tr-J SC.

Discussion. The presence of PAR associated to non-compact myelin regions (which constitute E-cadherin rich autotypic AJ/actin anchorage regions) and the co-alterations experienced by PAR and the actin cytoskeleton in epithelium and nerves, suggest that PAR may be a constitutive component of $A$ J/actin anchorage regions. Is PAR stabilizing the AJ-actin complexes? This question has strong implications in structural cell biology and cell signaling networks. Moreover, if PAR played a stabilizing role, such stabilization could participate in the physiological control of axonal branching.

PARP and PAR alterations exist in several neurodegenerative pathologies including Alzheimer's, Parkinson's and Hungtington's diseases. Conversely, PARP inhibition decreases PAR and promotes neurite 
outgrowth in cortical neurons in vitro. Coherently, the PARP inhibitor XAV939 improves myelination in vitro, ex vivo and in vivo. Until now such results have been interpreted in terms of nuclear PARP activity. Our results indicate for the first time the presence of PARylation in peripheral nerve fibers, in a healthy environment. Besides, we have evidenced a PARylation increase in Tr-J, suggesting that the involvement of cytoplasmic PARPs and PARylation in normal and neurodegenerative conditions should be reevaluated. 
4 Laura I. Lafon-Hughes ${ }^{1}$, Carlos J. Romeo ${ }^{2}$, Karina B. Cal ${ }^{2}$, Salomé C. Vilchez-Larrea ${ }^{3}$, José R. Sotelo $^{2}$, Gustavo A. Folle ${ }^{1}$, Silvia H. Fernández Villamil ${ }^{3,4}$, Alejandra E. Kun ${ }^{2,5}$.

$6{ }^{1}$ Departamento de Genética, Instituto de Investigaciones Biológicas Clemente Estable (IIBCE), 7 Montevideo, Uruguay.

$8 \quad 2$ Departamento de Proteínas y Ácidos Nucleicos, IIBCE, , Montevideo, Uruguay.

$9{ }^{3}$ Instituto de Investigaciones en Ingeniería Genética y Biología Molecular "Dr. Héctor N.

10 Torres", Consejo Nacional de Investigaciones Científicas y Técnicas, Buenos Aires, Argentina.

$11{ }^{4}$ Departamento de Química Biológica, Facultad de Farmacia y Bioquímica, Universidad de 12 Buenos Aires, Buenos Aires, Argentina.

$13{ }^{5}$ Departamento de Biología Celular y Molecular, Sección Bioquímica, Facultad de Ciencias, 14 Universidad de la República, , Montevideo, Uruguay.

15 Corresponding authors Alejandra E. Kun and Laura I. Lafon-Hughes

16 emails: akun@fcien.edu.uy, lauralafon2010@gmail.com 


\section{Abstract}

18 Background. Poly-ADP-ribose (PAR) is a polymer synthesized by poly-ADP-ribose polymerases (PARPs) as a postranslational protein modification and catabolized mainly by polyADP-ribose glycohydrolase (PARG). In spite of the existence of cytoplasmic PARPs and PARG, research has been focused on nuclear PARPs and PAR, demonstrating roles in the maintenance of chromatin architecture and the participation in DNA damage responses and transcriptional regulation. We have recently detected non-nuclear PAR structurally and functionally associated to the E-cadherin rich zonula adherens and the actin cytoskeleton of VERO epithelial cells. Myelinating Schwann cells are stabilized by E-cadherin rich autotypic adherens junctions. We wondered whether PAR would map to these regions. Besides, we have demonstrated an altered microfilament pattern in peripheral nerves of Trembler-J (Tr-J) model of CMT1-E. We hypothesized that cytoplasmic PAR would accompany such modified F-actin pattern.

Methods. Sibling WT and Tr-J mice sciatic nerves cryosections were subjected to immunohistofluorescence with anti-PAR antibodies. F-actin was detected with a phalloidin probe and DNA was counterstained with DAPI. Confocal image stacks were subjected to a colocalization highlighter and to semi-quantitative image analysis. Besides, antibody validation was carried.

Results. In the present work, we have shown for the first time the presence of PAR in sciatic nerves. Cytoplasmic PAR colocalized with F-actin at non-compact myelin regions in WT nerves. Moreover, in Tr-J, cytoplasmic PAR was augmented in close correlation with actin. In addition, nuclear PAR was detected in wild-type (WT) Schwann cells and was moderately increased in Tr-J Schwann cells.

Discussion. The presence of PAR associated to non-compact myelin regions (which constitute Ecadherin rich autotypic adherens junction/actin anchorage regions) and the co-alterations experienced by PAR and the actin cytoskeleton in epithelium and nerves, suggest that PAR may

42 be a constitutive component of adherens junction/actin anchorage regions. Is PAR stabilizing the 43 adherens junctions-actin complexes? This question has strong implications in structural cell 44 biology and cell signaling networks. Moreover, if PAR played a stabilizing role, such 45 stabilization could participate in the physiological control of axonal branching. 
46 PARP and PAR alterations exist in several neurodegenerative pathologies including Alzheimer's, 47 Parkinson's and Hungtington's diseases. Conversely, PARP inhibition decreases PAR and 48 promotes neurite outgrowth in cortical neurons in vitro. Coherently, the PARP inhibitor XAV939 49 improves myelination in vitro, ex vivo and in vivo. Until now such results have been interpreted in 50 terms of nuclear PARP activity. Our results indicate for the first time the presence of PARylation 51 in peripheral nerve fibers, in a healthy environment. Besides, we have evidenced a PARylation 52 increase in Tr-J, suggesting that the involvement of cytoplasmic PARPs and PARylation in 53 normal and neurodegenerative conditions should be re-evaluated. 


\section{Introduction}

55 Adherens junctions are protein complexes localized at intercellular junctions, characterized by the

56 existence of a link to the actin cytoskeleton at their cytoplasmic face. The central molecules in

57 these junctions are transmembrane proteins called cadherins. While extracellularly bound to a

58 neighbor identical molecule, cadherins are intracellularly attached to several proteins that allow

59 the anchorage of the actin microfilaments. In polarized epithelial cells, adherens junctions are

60 grouped. They describe a band across the lateral cell faces which encircles the cell, called the

61 zonula adherens or the adhesion belt, usually more basal than tight junctions (Alberts et al. 2002,

62 Meng \& Takeichi 2009). Epithelial adherens junctions functions are not only structural but also

63 regulatory, participating in cell signaling networks. For example, some adherens junctions

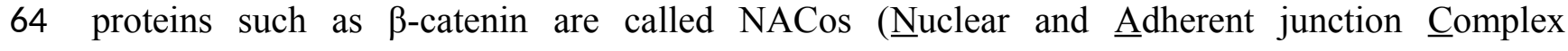

65 components). NACos can either localize at the adherens junctions or translocate to the nucleus

66 where they act as transcription factors, allowing the coordination of the loss of cellular adhesion

67 with cell rounding and mitosis entrance (Pérez-Moreno et al. 2003, Cerejido et al. 2004).

68 Clustered adherens junctions can also be found in other polarized cells. For example in the

69 vertebrate peripheral nervous system, each axon is surrounded by Schwann cells and an

70 extracellular matrix. These components altogether constitute the nervous fiber, characterized by

71 longitudinal as well as radial polarity. Myelinating Schwann cells wrap around the axons of

72 motor and sensory neurons to form the myelin sheath. E-cadherin autotypic junctions contribute

73 to the overall stability of the Schwann cell, being located in all the autotypic junctions regions,

74 namely the outer and inner mesaxons, the outer and inner loops, the paranodal regions and the

75 Schmidt-Lanterman incisures (Fannon et al. 1995, Poliak et al. 2002).

76 Interestingly, Trembler-J (Tr-J) mice harbour a punctual missense mutation in peripheral myelin

77 protein 22 (pmp-22) gene, constituting a model of hypomyelinating human peripheral neuropathy

78 Charcot-Marie-Tooth-1E (CMT1-E, Li et al. 2013; formerly classified as CMT1-A; Valentijn et

79 al. 1992). Tr-J mice display an increased number of Schwann cells (Robertson et al. 1997) as well

80 as structural alterations in Schmidt Lanterman incisures and paranodes of their sciatic nerve

81 fibers involving changes in autotypic adherens junctions components such as E-cadherin

82 (Devaux \& Scherer 2005). Moreover, actin microfilaments abundance and distribution in sciatic

83 nerve fibers are distorted (Kun et al. 2012a). 
84 Poly-ADP-ribose (PAR) is a linear or branched polymer of up to 400 ADP-ribose units. It is 85 synthesized by poly-ADP-ribose polymerases (PARPs) from $\mathrm{NAD}^{+}$as a postranslational protein 86 modification and catabolized mainly by the endo- or exo-glicosidic action of poly-ADP-ribose 87 glycohydrolase (PARG) (Virag \& Szabó 2002). PAR can in turn interact non-covalently with 88 different protein domains including PBZ (PAR binding zinc finger motif), WWE (with three 89 conserved residues -tryptophans and glutamate-) and Macrodomain (Leung 2014).

90 Excessive nuclear PARP and PAR occur in brains in the context of neurodegeneration for 91 example in Alzheimer's, Parkinson's and Hungtington's diseases (Love et al. 1999, Martire et al. 92 2015, Vis et al. 2005, Cardinale et al 2015). Besides, PAR quantity and/or PARP expression are 93 altered in several other pathologies (Strosznajder et al. 2000, Virag \& Szabó 2002, Strosznajder et al. 2005, Masutani et al. 2005, Lafon-Hughes et al. 2008, Cerboni et al. 2010, Strosznajder et al. 2012, Liu et al. 2014). Nevertheless, poly-ADP-ribosylation (PARylation) biology is still poorly understood.

Human PARP family has 17 members, 4 of which have PARylating activity. They are PARP-1, PARP-2, tankyrase-1 (TNKS-1) and tankyrase-2 (TNKS-2; Vyas et al. 2005). In spite of the fact that PARP-1 is the only member that is localized exclusively in the nucleus and PARG nuclear and cytoplasmic isoforms have been described, most PARylation studies are focalized in the cell nucleus. Nuclear PARPs regulate chromatin structure and somehow participate in nuclear networks regulating DNA replication, gene expression, DNA damage recognition and repair or telomere maintenance (Virag \& Szabó 2002). More recently, cytoplasmic roles of PARylation are being envisaged (Lehtio et al. 2013, Vyas et al. 2013). For example, in epithelial cells TNKS-1 maps not only to the nucleus but also to endoplasmic reticulum, Golgi apparatus, secretion vesicles, lisosomes or epithelial lateral membrane. Moreover, TNKS-1 is recruited from the cytoplasm to the epithelial lateral membrane upon formation of E-cadherin-based adherens junctions (Yeh et al. 2006). Adherens junctions proteins vinculin and catenin have been recovered as PARylated proteins in immunoprecipitation experiments (Gagné et al. 2008, 2012). Furthermore, VERO cells (green monkey renal epithelial cells) harbor a PAR belt associated to

111 the epithelial adhesion belt which is synthesized during the cell-cell adhesion process. If actin 112 polymerization is inhibited, the PAR belt is disaggregated. Conversely, if PAR belt synthesis is 113 inhibited by the TNKS inhibitor XAV939, the actin cytoskeleton, cell shape and cell adhesion are 114 altered, indicating that the PAR belt is structurally and functionally linked (directly or indirectly) 115 to the actin cytoskeleton (Lafon-Hughes et al. 2014). 
116 With the aim of contributing to understanding the biology of PARylation in the peripheral 117 nervous system, we tested the following three hypothesis. First, as PAR exists associated to

118 epithelial adherens junctions in VERO cells, it might be also found in peripheral nervous system 119 Schwann cell non-compact myelin which is rich in autotypic adherens junctions. Second, as PAR 120 is associated to the actin cytoskeleton in VERO cells and F-actin is highly increased in Tr-J 121 sciatic nerve fibers, PAR might be more abundant in Tr-J than WT nerves. Third, as nuclear PAR 122 increases have been described in central neuropathology, nuclear Schwann cell-PAR could be 123 increased in neurodegenerative Tr-J peripheral nerve fibers.

124 In the present work, we have used immunohistofluorescence (IHF) and confocal microscopy to 125 evidence for the first time the presence of PAR in Schmidt Lanterman incisures and paranode 126 regions in WT sciatic nerves. The non compact myelin regions where PAR and actin colocalized 127 mimicked E-cadherin well-described distribution. Besides, we demonstrated through filamentous 128 actin (F-actin) and PAR signals quantification on cytoplasmic axonal and Schwann cell domains 129 that Tr-J mice sciatic nerves have increased PAR.

\section{Materials and Methods}

131 Mice sciatic nerves

132 Institutional and national guidelines for the care and use of laboratory animals were followed. All 133 animal procedures were performed following the recommendations of the Committee of Ethics in 134 the Use of Animals (CEUA)-IIBCE, approved experimental protocols 011/11/2014 and 135 002/05/2016. Male 70 to 90 days old (P70-P90) wild-type (WT) and heterozygous mice carrying 136 a mutation in pmp-22 (Tr-J) from Jackson Laboratory (strain B6.D2-Pmp22 Tr-J/J) were killed by 137 cervical dislocation. Sciatic nerves dissection was promptly carried out in less than one minute 138 and followed by fixation through immersion in cold freshly prepared $3 \% \mathrm{w} / \mathrm{v}$ paraformaldehyde 139 (PFA) in PHEM buffer (60 mM Pipes, 25 mM Hepes, 10 mM EGTA, 2 mM magnesium chloride, $140 \mathrm{pH} \mathrm{7.2-7.6)} \mathrm{for} 1 \mathrm{~h}$. This procedure is known to grant a faster fixation than systemic descendent 141 perfusion in the singular case of mice sciatic nerves (Kun et al. 2012a,b). Then, nerves were 142 cryoprotected in sucrose/PHEM at $4^{\circ} \mathrm{C}$ (increasing concentrations along $24 \mathrm{~h}: 5 \%$ to $30 \% \mathrm{w} / \mathrm{v}$ ) 143 (Kun et al. 2012a,b). Next, tissue infiltration was done through progressive substitution (25\%, $14450 \%, 100 \%$ ) of sucrose/PHEM by Jung Tissue Freezing Medium (Leica 0201 08926). 145 Cryosections $(10 \mu \mathrm{m})$ were cut using a Cryostat (SLEE) and adhered to slides precoated with 146 chromic gelatin. Optimum results were obtained when overnight cryoprotection followed by 
147 infiltration, embedding, freezing and cutting were done within $30 \mathrm{~h}$. Cryosections were stored at

$148-20^{\circ} \mathrm{C}$ and immunostaining was started as soon as possible, in the following $24 \mathrm{~h}$.

149 Immunostaining with BD anti-PAR antibody

150 Before immunostaining, sciatic nerve sections were air-dried for $10 \mathrm{~min}$ at room temperature

151 (RT) and incubation chambers were delimitated on slides with nail polish. Sections were washed

152 in filtered PHEM (fPHEM), postfixed in $2 \% \mathrm{w} / \mathrm{v}$ PFA/fPHEM for $15 \mathrm{~min}$, washed in fPHEM,

153 and permeabilized in $0.1 \% \mathrm{v} / \mathrm{v}$ Triton-X100/fPHEM for $30 \mathrm{~min}$. Free aldehydes were blocked

154 with 1\% w/v sodium borohydride (NaBH4, Fluka 71320) in fPHEM (10 min). Then, tissue

155 sections were washed in PHEM and immersed for 30 min in blocking buffer [100 mM L-Lysine

156 (SIGMA L5501), 0.1\% w/v bovine seroalbumin (BSA, SIGMA A-2153) and 5\% v/v goat serum

157 in PHEM]. Sciatic nerve cryosections were incubated with 1:200 Becton Dickinson rabbit anti-

158 PAR (BD 551813) for $2 \mathrm{~h}$ at $37^{\circ} \mathrm{C}$ diluted in incubation buffer [200 mM L-lysine and $0.1 \% \mathrm{w} / \mathrm{v}$

159 BSA in PHEM]. After washing in PPHEM, sections were incubated for $1 \mathrm{~h}$ at RT in blocking

160 buffer with goat anti-rabbit secondary antibodies conjugated to Alexa 488 (Invitrogen A11034)

161 and an Alexa Fluor 546-phalloidin probe (Molecular Probes TM A22283) to evidence

162 filamentous actin (F-actin). Finally, after nuclear counterstaining with DAPI (1.5 $\mu \mathrm{g} / \mathrm{mL} / \mathrm{fPHEM}$;

163 Molecular Probes TM D21490), slides were rinsed in fPHEM, mounted in Vectashield (Vector

164 94010) or Prolong Gold (Molecular Probes P36930) and sealed with nail polish.

165 Controls without primary antibody were always run in parallel to check the specificity of the

166 detected signals. Moreover, to assure that specifically in sciatic nerves the antigen detected was

167 PAR, two complementary approaches were undertaken.

168 BD anti-PAR antibody validation in sciatic nerve. I: alternative anti-PAR antibody

169 ENZO BML-SA216 anti-PAR antibody was labelled using a kit (CFTM 488A) following the 170 manufacturer's instructions. Then, direct IHF was performed.

171 BD anti-PAR antibody validation in sciatic nerve. II: PAR digestion on fixed tissue sections

172 Sciatic nerve sections were subjected to digestion with recombinant human PARG (SIGMA 173 SRP8023 lot A00634/A, containing $2 \mu \mathrm{g}$ of PARG in $10 \mu \mathrm{L}$ buffer). PARG effect was studied 174 following ENZO recombinant PARG protocols (ALX-202-045-UC01), in a reaction buffer 175 essentially described by Ménard and Poirier (1987), used by Thomassin et al. (1990, 1992) and 


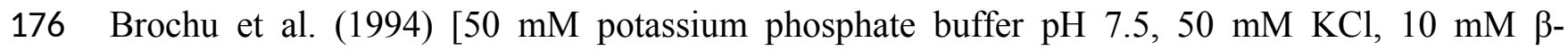

177 mercaptoethanol, 10\% v/v glycerol, $1 \mathrm{mM}$ DTT and 0.1\% v/v Triton-X100]. Fixed sciatic nerve

178 slices were rinsed in PARG reaction buffer and then incubated at RT with or without $50 \mathrm{nM}$

179 PARG in $100 \mu \mathrm{L}$ reaction buffer for $24 \mathrm{~h}$. Then, indirect IHF with BD anti-PAR antibody was

180 performed following the above described protocol.

181 Confocal microscopy and image analysis

182 Image stacks were collected with an OLYMPUS FV300 or a Leica TCS SP5 II confocal

183 microscope using 40x dry objectives (only overviews), a Plan Apo 60x/1.42 NA, a Plan Apo 63

$184 \mathrm{x} / 1.4$ NA or a Plan Apo 100x/1.4 NA oil immersion objectives, with or without digital zoom. To

185 assure signal specificity, original images were taken in the same conditions as reference images

186 of cells not labeled with primary antibodies, at the same confocal session.

187 Nerve cutting ends were skipped (either excluding them before cryoprotection or avoiding the

188 tips throughout image collection and quantification).

189 All images were processed and analyzed using Image $\mathrm{J}$ free software. Then data were exported to

190 Microsoft Excel (Office 2016 Home and Student Microsoft 79G-04351).

191 Olympus Fluoview images were opened using UCSD/Fluoview control plugin. LEICA (.lif) files 192 were extracted and saved as .tiff. The images used for illustrations were processed adjusting 193 brightness/contrast in parallel in WT, TrJ and without primary antibody images to avoid artifacts, 194 and finally smoothed (ratio1 Gaussian blur).

19516 animals were sacrificed to study sciatic nerves PARylation levels and distribution (14 of them 196 in 7 paired experiments including one WT and one Tr-J sibling). We evaluated qualitatively 7 197 experiments and quantitatively 3 experiments. Figshare links to see original microscopy stacks, 198 processed figures, ROIset examples, raw data, normalized data, summarized data and statistics 199 can be found at Table S1.

200 "Blind" quantification was impossible, given the differences in nuclei number, actin and PAR 201 distribution and fluorescence levels. These characteristics immediately revealed the genotype of 202 the sample being quantified.

203 Relative quantitation of F-actin and PAR was done on crude images using data from 3 204 independent experiments with paired animals (WT and Tr-J brothers). Images were taken under 
205 identical conditions for WT and Tr-J in each experiment, taking as a reference the control without 206 primary antibody.

207 To evaluate cytoplasmic relative contents, F-actin (red) and PAR (green) signal strength was 208 measured on at least 350 DAPI-negative regions of interest (ROIs) marked through the fiber 209 diameter of 12 stacks per experimental condition. The length of the ROI was normalized and 210 divided into 10 equal parts. In each experiment, the average relative intensity of actin and PAR 211 signals along the fiber diameter was calculated. Besides, assuming that the intervals from 0 to $21230 \%$ and from 70 to $100 \%$ correspond to Schwann cell (SC) regions whereas the range of 30 to $21370 \%$ corresponds to axon regions (Kun et al. 2012a), it was possible to calculate the average 214 relative intensity of F-actin and PAR in the axon. Then the average relative intensities of PAR and 215 actin in the axon were assigned the value 1 and used to normalize all the measurements. Finally, 216 normalized data from the three experiments were pooled. Mean intensities in arbitrary units 217 (relative to axon WT), standard deviations and standard errors were calculated for F-actin and 218 PAR signals in WT and Tr-J axons and SC. Statistical significance was tested using two-tailed 219 Student's t-test, with $\mathrm{p}<0.001$.

220 The gross anatomical differences between the WT and TrJ nerves could have skewed the signal 221 quantification. In order to exclude such a putative artifact, we present data (originally collected 222 with a different purpose in the context of Karina Cal's Master Thesis, 2017) showing that an 223 unrelated signal, quantified following the same methodology (on ROIs through fiber diameters) 224 did not increase. This signal corresponds to the cytoplasmic fraction of the octamer binding 225 transcription factor-6 (OCT6), which is a nucleocytoplasmic shuttling protein according to 226 Baranek et al. (2005).

227 To asses relative nuclear PAR contents, the ImageJ plugin "Intensity_Ratio_Nuclei_Cytoplasm" 228 was used. This allowed an automatic recognition of nuclear borders and areas. To avoid biases 229 due to differences in nuclear number or area, we measured the average nuclear PAR signal 230 intensity. This was done in at least 8 stacks per condition from 3 independent experiments. Again, 231 data were normalized according to mean nuclear PAR in each experiment and then were pooled. 232 Mean intensities in arbitrary units (relative to nuclear WT PAR), standard deviations and standard 233 errors were calculated. Statistical significance was tested using two-tailed Student's t-test, with $234 \mathrm{p}<0.001$. 
235 The "Colocalization Analysis/Colocalization Highlighter" ImageJ plugin (P. Bourdoncle, Institute

236 Jacques Monod, Service Imagerie, Paris, France) was used to highlight the cell regions where F-

237 actin and PAR colocalize. The user arbitrarily establishes a minimum threshold for each channel.

238 In this case, the threshold was experiment-dependent but WT, Tr-J and without primary antibody

239 images were processed in the same way. The program highlights (in yellow/orange) the pixels

240 where the intensity is above the threshold for both channels. Nothing was highlighted in the

241 absence of PAR primary antibody (black image); this was just an image processing control.

\section{Results}

243 WT mice sciatic nerves contained epitopes recognized by BD anti-PAR antibodies, particularly

244 evident in Schmidt Lanterman incisures, paranodes and outer loop regions

245 Nerve cryosections were subjected to indirect inmunohistofluorescence (IHF) with rabbit anti-

246 PAR antibody as well as F-actin detection with phalloidin-543 probe and nuclear counterstaining

247 with DAPI.

248 Throughout the work, figures illustrate fluorochrome signals corresponding to PAR (detected 249 with BD anti-PAR antibody) in green, F-actin in red and DAPI in blue. Fig. 1A,B shows single 250 confocal slices where PAR signals (absent in the control without primary antibody) can be seen.

251 For further analysis, colocalization was defined as the spatial overlap (in a single pixel) of two

252 signals (red and green, correspondent to F-actin and PAR channels), being each of the signals 253 above a certain intensity threshold (fixed arbitrarily in an experiment-specific way).

254 When pixels where F-actin and PAR signals colocalized were highlighted in yellowish/orange 255 color, appealing images were obtained. The F-actin-PAR colocalization regions (overviewed in 256 Fig. 1C) drew the known non-compact myelin regions in sciatic nerves where E-cadherin has 257 been localized, namely Schmidt Lanterman incisure (Fig. 1D-I), outer loops and paranodes (Fig. 258 1K-P). The observed distribution of $\mathrm{F}$ actin-PAR is in close agreement to the E-cadherin 259 distribution reported by Fannon and collaborators (see Figure 5 of Fannon et al. 1995; ).

260 F-actin increase in Tr-J mice sciatic nerves was paralleled by BD anti-PAR signal rise

261 Once PAR signal was detected in WT sciatic nerves, parallel experiments were carried in WT and 262 Tr-J siblings. As can be seen in Fig. 2 A-F, alterations in the distribution of PAR in Tr-J sciatic 263 nerves were observed. A careful quantification of F-actin and PAR signals along the fiber 
264 diameters in DAPI-negative (cytoplasmic) fiber regions was done (Fig. 2 G). Subsequently, a 265 previously established structural criteria (Kun et al. 2012a), stating that roughly the inner $40 \%$ of 266 the fiber diameter corresponds to the axon whereas the remnant outer region corresponds to the 267 Schwann cell, was adopted. This assumption allowed to affirm that both in Schwann cells (Fig. $2682 \mathrm{H}$ ) and in axonal domains (Fig. 2I), PARylation increased coarsely three to four times, like F269 actin, in Tr-J compared to WT nerves. Interestingly, an unrelated signal, corresponding to 270 cytoplasmic OCT6, quantified following the same methodology (on ROIs through fiber 271 diameters) did not increase (see Fig. S1), demonstrating that the observed elevation was not a 272 quantification artifact.

273 Fig. 3A-C shows a Tr-J sciatic nerve from the same experiment as the WT nerve in Fig. 1A-C. 274 Besides, image processing was exactly the same; therefore, it is comparison-prone. Fig. 3D-I, 275 comparable to Fig. 1N-P, shows that the axon is the main region where F-actin and PAR 276 colocalize in Tr-J sciatic nerves. An analogous phenomenon is observed in Fig. 3J-O, depicting 277 Tr-J Schmidt Lanterman incisure comparable to WT Schmidt Lanterman incisure in Fig. 1D and 278 1G-I. In conclusion, PAR distribution in Tr-J sciatic nerve was altered even in still identifiable 279 non-compact myelin regions.

280 Although this work was focused on cytoplasmic PARylation, nuclear PARylation was also 281 detected. The average PAR signal in the nuclear area in Tr-J sciatic nerves was increased 2.5 282 times in relation to WT average nuclear signal.

283 ENZO anti-PAR antibody but not Tulip clone H10 anti-PAR antibody reproduced the signals 284 obtained with BD anti-PAR antibody in WT and Tr-J sciatic nerves

285 ENZO mouse monoclonal antibody, raised against short to mid-PAR chains (2 to 50 units) 286 showed a clean signal after direct IHF (see Fig. S2). In fact, ENZO anti-PAR depicted clearly the 287 paranodes, Schmidt Lanterman incisure and outer loops (Fig. S2A,B) in WT sciatic nerves. An 288 overview shows again that Tr-J sciatic nerves, which harbor extra nuclei (Fig S2C vs F) as well 289 as F-actin increase (D vs G), do also depict PAR increase (E vs H). The same can be perceived 290 comparing I (WT) and J (Tr-J) merged channels images. Thus, ENZO anti-PAR antibody 291 qualitatively conduces to the same conclusions as BD anti-PAR antibody (and this has been 292 confirmed even quantitatively in nuclear WT vs Tr-J signals; see the Figshare link on Table S1). 
293 In contrast, Tulip H10-clone antibody, known to recognize preferentially long branched PAR 294 chains above 20 residues (Kawamitsu et al. 1984), showed no signal at all although it was 295 assayed both in WT and Tr-J sciatic nerves (data not shown).

296 BD anti-PAR signal was diminished after fixed sciatic nerves incubation in PARG.

297 PARG effect was studied in a PARG reaction buffer (PARG-buff) essentially described by 298 Ménard \& Poirier (1987) which was later modified by Thomassin et al. (1990), Brochu et al. 299 (1994) and has given rise to ENZO recipe. PARG-buff was the control against which the 300 digestion was evaluated.

301 To estimate an adequate PARG concentration, it was taken into account that a commercial PARG 302 kit (Trevigen 4682-096-K) uses $5 \mathrm{ng}$ of PARG in $100 \mu \mathrm{L}$ to degrade PAR associated to $20 \mu \mathrm{g}$ of 303 protein extract in $30 \mathrm{~min}$ at RT. Besides, $6.75 \mathrm{ng}$ of recombinant bovine PARG is enough to carry 304 a biochemical PARG degradation in an assay tube (see Fig. 6 from Meyer et al. 2007). Tissue 305 digestion requires higher enzymatic concentrations, sometimes orders of magnitude higher, than 306 biochemical "tube-reactions" . For example, an RNAse concentration of $20 \mu \mathrm{g} / \mathrm{mL}$ is used in 307 DNA extraction protocols (Sambrook \& Russell 2001) while a 500 times higher concentration $308(10 \mathrm{mg} / \mathrm{mL})$ has been used to digest tissue RNA in situ (Sotelo et al. 2013). Nevertheless, as the 309 amount of available enzyme was limited, escalating was not an option. Thus, $50 \mathrm{nM}$ PARG in $310100 \mu \mathrm{L}$ PARG-buff was finally used in a $24 \mathrm{~h}$ digestion protocol. PARG effect on sciatic nerve 311 fixed tissue sections was not homogeneous. We could observe tissue regions where nothing 312 happened coexisting with huge or intermediate PARG effects. By huge effects it is meant that 313 PAR signal reached $10 \%$ in digested tissue relative to non-digested one (see Fig. S2K vs $1 \mathrm{~L}$ ). A 314 clear difference could also be seen comparing non-digested with digested tissue in Fig. S2K vs L, $315 \mathrm{~N}$ vs $\mathrm{P}$ or $\mathrm{R}$ vs T. By intermediate effects it is meant partial digestion, like the PAR "cloud" 316 observed in Fig. S2M. The reached PAR digestion in our borderline experimental conditions 317 (PARG concentration in the same order as in tube biochemical reactions, small digestion volume 318 and uneven tissue surface) was a convincing result.

319 Interestingly, the F-actin levels seemed to diminish together with PAR levels at the digested 320 regions (Fig. S2.N-U). 
321 To sum up, the evidence proves the PAR identity of IHF PAR signals, validating the biological 322 findings, namely: (1) PAR colocalizes with with F-actin at non-compact myelin regions; (2) in a

323 Charcot-Marie-Tooth demyelinating disease model in which sciatic nerves present an excess of 324 F-actin, accompanied by E-cadherin delocalization (Kun et al. 2012a, Devaux \& Scherer 2005),

325 detected PAR was excessive and was delocalized too; (3) nuclear PAR was detectable in WT and 326 moderately increased in Tr-J sciatic nerve Schwann cells.

\section{Discussion}

328 This study represents the first report of PAR presence and distribution in the peripheral nerve 329 system. We demonstrated that WT mice sciatic nerves contained nuclear PAR as well as 330 cytoplasmic PAR. The latter colocalized with F-actin at E-cadherin rich non-compact myelin 331 regions including Schmidt Lanterman incisures and paranodes.

332 Like in VERO epithelial belt, PAR was detected with anti-PAR antibodies that are presumed to 333 recognize short to middle chain length polymer (BD and ENZO anti-PAR antibodies). The 334 finding of PAR associated to E-cadherin rich/actin anchorage regions in two different biological 335 systems (a monkey epithelial cell line and mice nerves) suggests that PARylation may be 336 involved in adherens junction biology. These findings have deep implications in terms of 337 junctional structures and cell signaling.

338 It has been proposed that inhibition of axonal regeneration by myelin after injure is an aberrant 339 effect of an otherwise physiological inhibitory function (Shen et al. 1998). Axon branching 340 inhibition is essential for the structural economy of the peripheral nervous system, especially for 341 long axons. One key mediator of these functions would be myelin-associated glycoprotein 342 (MAG; Mukhopadhyay et al. 1994, Schafer et al. 1996, Shen et al. 1998). MAG is mainly found 343 at the adaxonal side of the Schwann cell membrane and non-compact myelin regions rich in 344 autotypic adherens junctions including Schmidt Lanterman inscisures and paranodes (Martini and 345 Schachner 1988, Ghabriel and Allt 1980, Erb et al. 2006).

346 In WT mice we observed PAR at non-compact myelin regions. Besides, PAR has been involved 347 in axonal regeneration inhibition (Brochier et al. 2015). Therefore, an hypothesis can be raised 348 regarding the putative physiological role of the observed PAR in preventing axonal branching.

349 Through image quantification of immunohistofluorescent images, we have also shown that Tr-J 350 sciatic nerve Schwann cells harbored excessive nuclear PAR. Interestingly, excessive nuclear 
351 PARP and PAR had already been reported in brains in the context of neurodegeneration for 352 example in Alzheimer's, Parkinson's and Hungtington's diseases (Love et al. 1999, Martire et al. 353 2015, Vis et al. 2005, Cardinale et al. 2015). In fact, PARP-1-/- mice confirm the protective role 354 of PARP-1 deficit towards injure induced by A $\beta$ injections (that mimic Alzheimer's disease), 355 MPTP or 6-OHDA (used to induce Parkinson-like symptoms). Thus, the current prevailing 356 interpretations are that different injures induce PARP-1 overactivation leading to cell death by 357 energy shortage due to $\mathrm{NAD}^{+}$overconsumption and/or that DNA repair alterations (which may 358 involve PARP-1 signaling) represent a common denominator in neurodegeneration (Martire et al. 359 2015, Ross and Truant 2016). However, in Tr-J, the absence of apoptotic morphology or long 360 branched polymer (recognized by H10 clone anti-PAR antibody) suggest that the excessive 361 nuclear PAR was not associated to a DNA repair response.

362 Our current work underscores the existence of a comparable increase of cytoplasmic PAR in a 363 Charcot-Marie-Tooth model. Interestingly, the first scientific work that communicates an increase 364 in human brain poly(ADP-ribosyl)ation with neurodegeneration (Love et al. 1999) is focused on 365 the strong nuclear PARP and PAR signal in Alzheimer's disease patients brains. Such work does 366 also show the existence of cytoplasmic PARP and PAR immunoreactivity, which is not further 367 remarked. Other authors measure a significant PARP activity increase in hippocampus 368 homogenates in contexts of excitotoxicity or amyloid beta peptide presence or, conversely, study 369 the effect of PARP inhibition with 3-aminobenzamide (3-AB) (Strosznajder et al. 2000, 2005). 370 They also arrive to the conclusion that PARP has a central role in neurodegeneration. We agree on 371 that conclusion but in our opinion it has to be emphasized that neither PARP activity 372 measurements nor PARP inhibition with 3-AB are evidencing exclusively nuclear PARP or 373 PARP-1 activity. These results could be reflecting the activity and the role of a pool of PARPs 374 (including PARP-1, PARP-2, TNKS-1 and TNKS-2), some of which are cytoplasmic.

375 In contrast to WT, Tr-J sciatic nerve axons harbor a strong PAR signal. PAR presence in 376 peripheral axons resembles the work of Brochier et al. (2015) in the central nervous system, since 377 they detect PAR in crushed optic nerve axons. Of note, we did not see any obvious difference in 378 PAR signal intensity at the nerve cutting ends (we skipped them for quantification purposes just 379 in case). Brochier et al. (2015) do also demonstrate that axonal growth inhibiting signals such as 380 Nogo, MAG or astrocyte-produced chondroitin sulfate proteoglycans (CSPGs), induce neuronal 381 PAR accumulation in primary cortical neurons. Likewise, the increase of MAG expression in the 
382 peripheral nervous system under neurodegenerative conditions (Kinter et al. 2013) might be 383 related to the high levels of PAR in the Tr-J nerve fibers.

384 About $80 \%$ of the basal PAR pool is conserved in PARP-1 ${ }^{-/}$primary cortical neurons (Brochier et 385 al. 2015). In contrast, the induced PAR increase depends on PARP-1 activity. PARP-1 is a nuclear 386 enzyme; just a small PARP-1 fraction has rarely been localized in the cytoplasm of cancer cells 387 (Donizy et al. 2014). Therefore, in Brochier et al. experiments, either neuronal PARP-1 can 388 somehow reach the cytoplasm under certain conditions or PARP-1 is part of a signalling cascade 389 that activates a cytoplasmic PARP that in turn synthesizes the cytoplasmic PAR. Interestingly, the 390 PARP inhibitors which restore the axonal growth that is diminished by Nogo, MAG or CSPGs 391 are not PARP-1 specific. Moreover, according to the same authors (Brochier et al. 2015), in a 392 microfluidic-based culture platform, most growth rate restoration by the PARP inhibitor is 393 achieved through PJ34 administration in the axonal compartment, sugesting that most PJ34 effect 394 can be reached through the inhibition of a cytoplasmic PARP. No increase in axonal regeneration 395 nor improvement in motor function recovery were observed after optic nerve crush or dorsal 396 hemisection of the spinal cord, in PARP $-1^{-/-}$mice or after systemic administration of the specific 397 PARP-1 inhibitor velaparib (Wang et al. 2016).

398 Disturbances in PMP22 are associated with abnormal myelination in a range of inherited 399 peripheral neuropathies both in mice and humans (Robertson et al. 1997). PMP22 is critical for 400 actin-mediated cellular functions (Lee et al. 2014). Interestingly, actin microfilaments assembly 401 and disassembly is essential during myelin sheath formation in the peripheral and central nervous 402 system (Park \& Feltri 2011, Feltri et al. 2008, Nawaz et al. 2015, Zuchero et al. 2015). A 403 lamellipodia-like structure driving myelin wrapping has been described in peripheral and central 404 myelination process (Salzer 2012, Feltri et al. 2015, Nawaz et al. 2015, Zuchero et al. 2015). 405 Besides, PMP22-deficient nerves depict early abnormal junctions and permeability of myelin 406 (Guo et al. 2014). Tr-J mice sciatic nerve has marked alterations in junctional proteins including 407 delocalized E-cadherin (Devaux \& Scherer 2005). In turn, cumulative evidence indicates that 408 adherens junctions proteins (E-cadherin, catenins, vinculin), some of which can act as NACos, 409 may play a significant role in the myelination process (Tricaud et al. 2005, Perrin-Tricaud et al. 410 2007, Ye et al. 2009, Peng et al. 2010, Beppu et al. 2015, Basak et al. 2015). For example, E411 cadherin enhances neuroregulin-1 (NRG1) signaling and promotes Schwann cell myelination 412 (Basak et al. 2015). It has to be emphasized that NRG1 is one of the major and best characterized 413 extrinsic signals that control myelination (Salzer 2015). Conversely, an aberrant localization of E- 
414 cadherin can be a potent inhibitor of $\mathrm{Wnt} / \beta$-catenin ( $\mathrm{Su}$ et al. 2015) and in mammalian CNS, 415 dysregulation of the Wnt pathway inhibits timely myelination (Fancy et al. 2009, Dai et al. 2014).

416 Considering our previous results regarding the actin cytoskeleton/PAR belt dialogue in VERO 417 cells (Lafon-Hughes et al. 2014) and the highly increased actin observed in the Tr-J sciatic nerve 418 (Kun et al. 2012a), the observed PAR increase was an expected result. Moreover, PAR-digested 419 regions seemed to harbor lower F-actin signals, suggesting that PAR might be somehow 420 participating in the holding or anchorage of at least part of the F-actin cytoskeleton network. In 421 the nerves, like in VERO cells, the actin-PAR connection seems to be active.

422 We don't know if PARylation alterations are upstream actin and E-cadherin modifications. It is 423 even unknown which proteins are PARylated in the sciatic nerve. Looking for putative 424 cytoplasmic PARylation candidates -adherens junctions/actin anchorage proteins or 425 microfilaments components-, we have inspected the macrodomain-recognized ADP-ribosylome 426 of liver epithelium; catenin (CTNNA 3), vinculin, and $\beta$-actin are in the list of affinity-enriched 427 proteins. As the macrodomain recognizes MAR and in some cases PAR (Martello et al. 2016), the 428 method does not differentiate mono-ADP-rybosylated (MARylated) from PARylated proteins. 429 Interestingly, this data fit with another proteomics work showing that in human embryonic kidney 430 cells (HEK 293; Gagné at al. 2012), vinculin and catenin ( $\beta$-catenin) were recovered in the pool 431 of proteins bound to a catalytically inactive GFP-PARG or "PARG-DEAD domain" (indicating 432 their probable PARylation or association to PARG) but not immunoprecipitated with clone H10 433 antibody (indicating that they do not bind long branched chains). Actin was not recovered in non434 stimulated cells. Instead, $\beta$-actin was enriched in PARylated complexes in MNNG-treated cells, 435 indicating its participation in the responses induced by this alkylating agent (Gagné et al. 2012). 436 In our knowledge, a single group claimed that actin is "the unique endogenous acceptor of PAR" 437 in an Octopus brain cytoplasmic subcellular fraction (De Maio et al. 2013).

438 Monomeric G-actin has been long-wide recognized as a MARylating target of bacterial toxins, 439 shifting the equilibrium towards microfilaments disassembly (De Maio et al. 2013). Coherently, 440 the knockdown of PARP-14, which harbors MARylating activity (Vyas et al. 2005) and is a focal 441 adhesion protein, results in cells that are unable to retract protrussions efficienty, generating 442 highly elongated extensions (Vyas et al. 2013). The knockdown of the macrodomain-containing 443 enzymatically-inactive PARP-9 results in abnormal membrane blebbing in the absence of typical 444 apoptotic nuclear DNA hypercondensation (Vyas et al. 2013), suggesting that PARP-9 is also 
445 involved in actin cytoskeleton dynamics. As PARP-9 is enzymatically inactive and PARP-14 has 446 just MARylating activity (Vyas et al. 2005), neither of them could be responsible for the synthesis 447 of the PAR that we observed in mice sciatic nerves. Nevertheless, the existence of some sort of 448 regulatory interaction among PARP-9 or PARP-14 and the PARP responsible for PAR synthesis 449 cannot be discarded.

450 The analogy with epithelial cells (Yeh et al. 2006, Lafon-Hughes et al 2014), points to TNKS 451 involvement in cytoplasmic PARylation. Mice deficient in either one TNKS are viable but 452 deficiency of both TNKS results in embryonic lethality, demonstrating that TNKS are essential 453 but at least partially redundant (Chiang et al. 2008). Accordingly, TNKS-2 knockdown cells 454 display no detectable phenotype whereas TNKS-1 knockdown cells display mitotic defects and 455 diminished viability (Vyas et al. 2013), probably preventing further dissection of the molecular 456 mechanisms involved.

457 Interestingly, a study on structural basis and sequence rules for substrate recognition by TNKS 458 identifies a TNKS-binding motif (Guettler et al. 2011) which is present in vinculin and catenin 459 but not in $\beta$-actin.

460 TNKS is necessary for canonical Wnt signaling (Kartner et al. 2010). TNKS inhibition induces 461 axin stabilization and blocks Wnt signaling (Bao et al. 2012). Besides, axin has been identified as 462 a regulatory and therapeutic target in newborn brain injury and remyelination (Fancy et al. 2011). 463 Moreover, anti-TNKS weapons promote myelination (Casaccia 2012). To be more precise, the 464 TNKS inhibitor XAV939 accelerates oligodendrocyte progenitor differentiation in cell cultures, 465 improves myelination and remyelination (following hypoxia or lysolecithin) in ex vivo mouse 466 cerebellar slice cultures and diminishes the demyelinating effects of lysolecithin in mice spinal 467 cord in vivo (Fancy et al. 2011). It has been demonstrated that the TNKS PARylation target 468 molecule axin is involved. It is likely that some of the adherens junctions/actin anchorage 469 proteins are PARylation targets too, acting in concert with axin to coordinate cell adhesion and 470 migration with differentiation.

471 Together our findings highlight the presence of PAR in specific regions of peripheral nerves and 472 its increase in Tr-J, opening a window to further explore the possible roles of cytoplasmic PAR 473 associated to adherens junctions/actin cytoskeleton in the whole nervous system. The advances in 474 this field are expected to contribute in the future to a more precise design of therapies, not only 
475 for CMT patients, which represent 1 every 2500 persons in the general population (Li at al. 476 2013), but also for other neurodegenerative disease patients.

\section{Conclusions}

478 - PAR was detected in mice sciatic nerves, colocalizing with F-actin at non-compact myelin 479 regions of peripheral nerve fibers which are rich in adherens junctions. The existence of $480 \quad$ PAR in adherens junctions regions in systems as divergent as Schwann cells and VERO 481 epithelial cells suggests that PAR may be a previously overlooked inherent component of the E-cadherin rich adherens junctions.

- PAR was in excess in a Charcot-Marie-Tooth demyelinating disease model in which sciatic nerves present an excess of F-actin. Like in VERO cells, our result argue in favor of a structural (direct or indirect) connection of PAR with F-actin that deserves further investigation.

- Nuclear PAR was present in WT and moderately increased in Tr-J Schwann cells, putatively affecting chromatin structure and functions.

\section{Acknowledgements}

490

We kindly acknowledge Archana Dhasarathy (University of North Dakota, USA) for the initial aliquots of ENZO anti-PAR antibody, Mariana Di Domenico (LEICA technician at Facultad de

Medicina) for technical advice and Martín Breijo, Mariela Santos (URBE Facultad de Medicina), Carmen Pérez (Rodents Bioterio, IIBCE) for animal handling and maintenance and Sergei Nechaev (University of North Dakota, USA), for manuscript critical reading.

\section{References}

Alberts B, Johnson A, Lewis J, Raff M, Roberts K, Walter P. 2002. Molecular Biology of the cell. Garland Science $\left(4^{\text {th }}\right.$ Ed), New York, USA.

Bao R, Christova T, Song S, Angers S, Yan X, Attisano L. 2012. Inhibition of tankyrases induces axin stabilization and blocks Wnt signaling in breast cancer cells. PLoS ONE 7:(11):e48670. doi: 10.1371/journal.pone.0048670

Baranek C, Sock E, Wegner M. 2005. The POU protein Oct-6 is a nucleocytoplasmic shuttling protein. Nucleic Acids Research 33(19):6277-6286. 
503 Basak S, Desai DJ, Rho E, Ramos R, Maurel P, Kim HA. 2015. E-cadherin enhances neuregulin

504 signaling and promotes Schwann cell myelination. Glia 63:1522-1536.

505 Beppu M, Sawai S, Satoh M, Mori M, Kazami T, Masawa S, Shibuya K, Ishibashi M, Sogawa K,

506 Kado S, Kodera Y, Nomura F, Kuwabara S. 2015. Autoantibodies against vinculin in patients

507 with chronic inflammatory demyelinating polyneuropathy. Journal of Neuroimmunology 287:9-

50815.

509 Brochier C, Jones JI, Willis DE, Langley B. 2015. Poly(ADP-ribose) polymerase 1 is a novel

510 target to promote axonal regeneration. Proceedings of the National Academy of Sciences (USA)

511 112(49):15220-15225.

512 Brochu G, Shah GM, Poirier GG. 1994. Purification of poly(ADP-ribose) glycohydrolase and 513 detection of its isoforms by a zymogram following one or two-dimensional electrophoresis. 514 Analytical Biochemistry 218:265-272.

515 Cal K. 2017. La neurodegeneración crónica Trembler J causa un desorden global del 516 citoesqueleto de actina en la fibra nerviosa periférica. Master Thesis. PEDECIBA Program, 517 Faculty of Sciences, University of the Republic (Uruguay).

518 Cardinale A, Paldino E, Giampà C, Bernardi G, Fusco FR. 2015. PARP-1 Inhibition Is 519 Neuroprotective in the R6/2 Mouse Model of Huntington's Disease. PLoS ONE 10(8):e0134482. 520 doi: 10.1371/journal.pone.0134482

521 Casaccia P. 2012. Anti-tankyrase weapons promote myelination. Nature Neuroscience 14(8):945522947.

523 Cerboni B, Di Stefano A, Micheli V, Morozzi G, Pompucci G, Sestini S. 2010. PARP activity and 524 NAD concentration in PMC from patients affected by systemic sclerosis and lupus 525 erythematosus. Nucleosides, Nucleotides and Nucleic Acids 29:471-475.

526 Cerejido M, Contreras RG, Shoshani L. 2004. Cell adhesion, polarity and epithelia in the dawn of 527 metazoans. Physiological Reviews 84(4):1229-1262.

528 Chiang YJ, Hsiao SJ, Yver D, Cushman SW, Tessarollo L, Smith S, Hodes RJ. 2008. Tankyrase 1 529 and Tankyrase 2 are essential but redundant for mouse embryonic development. PLoS ONE 530 3(7):e2639. doi: 10.1371/journal.pone.0002639. 
531 Dai ZM, Sun S, Wang C, Huang H, Zhang Z, Lu QR, Qiu M. 2014. Stage-specific regulation of 532 oligodendrocyte development by Wnt/ $\beta$-catenin signaling. The Journal of Neuroscience 533 34(25):8647-8673.

534 De Maio A, Natale E, Rotondo S, Di Cosmo A, Faraone-Menella MR. 2013. Vault poly(ADP535 ribose) polymerase in the Octopus vulgaris brain: a regulatory factor of actin polymerization 536 dynamics. Comparative Biochemistry and Physiology B 166:40-47.

537 Devaux JJ, Scherer SS. 2005. Altered ion channels in an animal model of Charcot-Marie-Tooth 538 disease type 1A. The Journal of Neuroscience 25(6):1470-1480.

539 Donizy P, Pietrzyk G, Halon A, Kozyra C, Gansukh T, Lage H, Surowiak P, Matkowski R. 2014. 540 Nuclear-cytoplasmic PARP-1 expression as an unfavorable prognostic marker in lymph node 541 negative early breast cancer: 15-year follow-up. Oncology Reports 31(4):1777-1787. doi: 542 10.3892/or.2014.3024.

543 Erb M, Flueck B, Kern F, Erne B, Steck AJ, Schaeren-Wiemers N. 2006. Unraveling the 544 differential expression of the two isoforms of myelin-associated glycoprotein in a mouse 545 expressing GFP-tagged S-MAG specifically regulated and targeted into the different myelin 546 compartments. Molecular and Cellular Neuroscience 31(4):613-627.

547 Fancy SP, Harrington EP, Yuen TJ, Silbereis JC, Zhao C, Baranzini SE, Bruce CC, Otero JJ, 548 Huang EJ, Nusse R, Franklin RJ, Rowitch DH .2011. Axin2 as a regulatory and therapeutic target 549 in newborn brain injury and remyelination. Nature Neuroscience 14(8):1009-1016.

550 Fancy SP, Baranzini SE, Zhao C, Yuk DI, Irvene KA, Kaing S, Sanai N, Franklin RJ, Rowitch 551 DH. 2009. Dysregulation of the Wnt pathway inhibits timely myelination and remyelination in 552 mammalian CNS. Genes \& Development 23(13):1571-1585.

553 Fannon AM, Sherman DL, Ilyna-Gragerova G, Brophy PJ, Friedrich VL, Colman DR .1995.

554 Novel E-cadherin-mediated adhesion in peripheral nerve: Schwann cell architecture is stabilized 555 by autotypic adherens junctions. The Journal of Cell Biology 129(1):189-202.

556 Feltri ML, Poitelon Y, Previtali SC . 2015. How Schwann cells sort axons: new concepts. 557 Neuroscientist 22(3): 252-265. 
558 Feltri ML, Suter U, Relvas JB. 2008. The Function of RhoGTPases in Axon Ensheathment and 559 Myelination. Glia 56:1508-1517.

560 Gagné JP, Isabelle M, Lo KS, Bourassa S, Hendzel MJ, Dawson VL, Dawson TM, Poirier GG. 561 2008. Proteome-wide identification of poly(ADP-ribose) binding proteins and poly(ADP-ribose)562 associated protein complexes. Nucleic Acids Research 36:6959-6976.

563 Gagné JP, Pic E, Isabelle M, Krietsch J, Ethier C, Paquet E, Kelly I, Boutin M, Moon KM, Foster 564 LJ, Poirier GG. 2012. Quantitative proteomics profiling of the poly(ADP-ribose)-related response 565 to genotoxic stress. Nucleic Acids Research 40:7788-7805.

566 Ghabriel MN, Allt G. 1980. Schmidt-Lanterman Incisures. I. A quantitative teased fibre study of 567 remyelinating peripheral nerve fibres. Acta Neuropathologica 52(2):85-95.

568 Guettler S, LaRose J, Petsalaki E, Gish G, Scotter A, Pawson T, Rottapei R, Sicheri F. 2011. 569 Structural basis and sequence rules for substrate recognition by tankyrase explain the base for 570 cherubism disease. Cell 147:1340-1354.

571 Guo JS, Wang LM, Zhang Y, Wu JW, Arpag S, Hu B, Imhof BA, Tian XX, Carter BD, Suter U, 572 Li J. 2014. Abnormal Myelin Junctions and Permeability in PMP22 Deficient Nerves; Annals of 573 Neurology 75 (2):255-265.

574 Kartner CM, Merkel CE, Dodge M, Ma Z, Lu J, Chen C, Lum J, Carroll TJ. 2010. Tankyrase is 575 necessary for canonical Wnt signaling during kidney development. Developmental Dynamics 576 239:2014-2023.

577 Kawamitsu H, Hoshino H, Okada H, Momoi H, Sugimura T. 1984. Monoclonal antibodies to 578 poly (adenosine diphosphate ribose) recognize different structures. Biochemistry 23:3771-3777.

579 Kinter J, Lazzati T, Schmid D, Zeis T, Erne B, Lützelschwab R, Steck AJ, Pareyson D, Peles E, 580 Schaeren-Wiemers N. 2013. An essential role of MAG in mediating axon-myelin attachment in 581 Charcot-Marie-Tooth 1A disease. Neurobiology of Disease 49:221-231. doi: 582 10.1016/j.nbd.2012.08.009.

583 Kun A, Canclini L, Rosso G, Bresque M, Romeo C, Hanusz A, Cal K, Calliari A, Sotelo-Silveira 584 JR, Sotelo JR. 2012a. F-actin distribution at nodes of Ranvier and Schmidt-Lanterman incisures 585 in mammalian sciatic nerves. Cytoskeleton 69:486-495. 
586 Kun A, Rosso G, Canclini L, Bresque M, Romeo C, Cal K, Calliari A, Hanusz A, Sotelo-Silveira 587 J, Sotelo JR. 2012b. The Swann cell-axon link in normal condition or neurodegenerative 588 diseases: an immunocytochemical approach. In: Deghani $\mathrm{H}$, ed. Applications of 589 immunocytochemistry. INTECH, Croatia, 249-266.

590 Lafon-Hughes L, Di Tomaso MV, Méndez-Acuña L, Martínez-Lopez W. 2008. Chromatin591 remodelling mechanisms in cancer. Mutation Research 658:191-214.

592 Lafon-Hughes L, Vilchez Larrea SC, Kun A, Fernández-Villamil S. 2014. VERO cells harbor a 593 poly-ADP-ribose belt partnering their epithelial adhesion belt. PeerJ 10.7717/peerj.617.

594 Lee S, Amici S, Tavori H, Zeng WM, Freeland S, Fazio S, Notterpeck L. 2014. PMP22 is critical 595 for actin-mediated cellular functions for establishing lipid rafts. The Journal of Neuroscience 596 34(48): 16140-16152.

597 Lehtio L, Chi NW, Krauss S. 2013. Tankyrases as drug targets. The FEBS Journal 280:35765983593.

599 Leung AKL. 2014. Poly(ADP-ribose): an organizer of cellular architecture. The Journal of Cell 600 Biology 205(5):613-619.

601 Li J, Parker B, Martyn C, Natarajan C, Guo J. 2013. The PMP22 gene and its related diseases. 602 Molecular Neurobiology 47(2):673-698.

603 Liu C, Srihari S, Cao KA, Chenevix-Trench G, Simpson PT, Ragan MA, Khanna KK. 2014. A 604 fine-scale dissection of the DNA double-strand break repair machinery and its implications for 605 breast cancer therapy. Nucleic Acids Research 42:6106-6127.

606 Love S, Barber R, Wilcock G. 1999. Increased poly(ADP-ribosyl)ation of nuclear proteins in 607 Alzheimer's disease. Brain 122:247-253.

608 Martello R, Leuotert M, Jungmichel S, Bilan V, Larsen SC, Young C, Hottiger MO, Nielsen ML. 609 2016. Proteome-wide identification of the endogenous ADP-ribosylome of mammalian cells and 610 tissue. Nature Communications 7:12917. doi 10.1038/ncomms12917.

611 Martini R, Schachner M. 1988. Immunoelectron microscopic localization of neural cell adhesion 612 molecules (L1, N-CAM, and myelin-associated glycoprotein) in regenerating adult mouse sciatic 613 nerve. The Journal of Cell Biology 106(5):1735-1746. 
614 Martire S, Mosca L, d'Erme M. 2015. PARP-1 involvement in neurodegeneration: a focus on 615 Alzheimer's and Parkinson's diseases. Mechanisms of Ageing and Development 146:53-64.

616 Masutani M, Nakagama H, Sugimura T. 2005. Poly(ADP-ribosyl)ation in relation to cancer and 617 autoimmune disease. Cell and Molecular Life Sciences 62:769-783.

618 Ménard L, Poirier GG. 1987. Rapid assay of poly(ADP-ribose) glycohydrolase. Biochemistry 619 and Cell Biology 65:668-673.

620 Meng W, Takeichi M. 2009. Adherens junction: molecular architecture and regulation. Cold 621 Spring Harbor Perspectives in Biology:1a002899. doi: 10.1101/cshperspect.a002899

622 Meyer RG, Meyer-Ficca ML, Whatcott CJ, Jacobson EL, Jacobson MK. 2007. Two small 623 enzyme isoforms mediate mammalian mitochondrial poly(ADP-ribose) glycohydrolase (PARG) 624 activity. Experimental Cell Research 313(13):2920-2936.

625 Mukhopadhyay G, Doherty P, Walsh FS, Crocker PR, Filbin MT. 1994. A novel role for myelin626 associated glycoprotein as an inhibitor of axonal regeneration. Neuron 3:757-767.

627 Nawaz S, Sánchez P, Schmitt S, Snadeiro N, Mitkovski M, Velte C, Brückner BR, Alexopoulos 628 L, Czopka T, Jung SY, Rhee JS, Janshoff A, Witke W, Schaap IA, Lyons DA, Simons M. 2015. 629 Actin filament turnover drives leading edge growth during myelin sheat formation in the central 630 nervous system. Developmental Cell 34(2):139-151.

631 Park HT, Feltri ML. 2011. Rac1 GTPase controls myelination and demyelination. 632 BioArchitecture 1(3):110-113.

633 Peng X, Cuff LE, Lawton CD, De Mali KA. 2010. Vinculin regulates cell surface E-cadherin 634 expression by binding to $\beta$-catenin. Journal of Cell Science 123(4):567-577.

635 Pérez-Moreno M, Jamora C, Fuchs E. 2003. Sticky Business. Orchestrating cellular signals at 636 adherens junctions. Cell 112(4):535-548.

637 Perrin-Tricaud C, Rutishauser U, Tricaud N. 2007. P120 catenin is required for thickening of 638 Schwann cell myelin. Molecular and Cellular Neuroscience 35(1):120-129. 
639 Poliak S, Matlis S, Ullmer C, Scherer SS, Peles E. 2002. Distinct claudins and associated PDZ 640 proteins form different autotypic tight junctions in myelinating Schwann cells. The Journal of 641 Cell Biology 159(2):361-371.

642 Robertson AM, King RH, Muddle JR, Thomas PK. 1997. Abnormal Schwann cell/axon 643 interactions in the Trembler-J mouse. Journal of Anatomy 190(3):423-432.

644 Ross CA, Truant R. 2016. DNA repair. A unifying mechanism in neurodegeneration. Nature 645 541(34-35):7635. doi:10.1038/nature21107

646 Salzer JL. 2012. Axonal regulation of Schwann cell ensheatment and myelination. Journal of the 647 Peripheral Nervous System 17(3):14-19. doi: 0.1111/j.1529-8027.2012.00425.x.

648 Salzer JL. 2015. Schwann cell myelination. Cold Spring Harbor Perspectives in Biology: 649 a020529. doi: 10.1101/cshperspect.a020529

650 Sambrook JF, Russell DW. 2001. Molecular Cloning: a laboratory manual. CSHL, New York, 651 USA.

652 Schafer M, Fruttiger M, Montag D, Schachner M, Martini R. 1996. Disruption of the gene for the 653 myelin-associated glycoprotein improves axonal regrowth in C57BL/Wld mice. Neuron 16:11076541113.

655 Shen YJ, DeBellard ME, Salzer JL, Roder J, Filbin MT. 1998. Myelin-associated glycoprotein in 656 myelin and expressed by Schwann cells inhibits axonal regeneration and branching. Molecular 657 and Cellular Neuroscience 12(1-2):79-91.

658 Sotelo JR, Canclini L, Kun A, Sotelo-Silveira JR, Xu L, Wallrabe H, Calliari A, Rosso G, Cal K, 659 Mercer JA. 2013. Myosin-Va-Dependent Cell-To-Cell Transfer of RNA from Schwann Cells to 660 Axons. PLoS ONE 8(4):e61905. doi: 10.1371/journal.pone.0061905

661 Strosznajder JB, Jesko H, Strosznajder RP. 2000. Effect of amyloid beta peptide on poly(ADP662 ribose) polymerase activity in adult and aged rat hippocampus. Acta Biochimica Polonica 663 47(3):847-854.

664 Strosznajder RP, Jesko H, Zambrzycka A. 2005. Poly (ADP-ribose) polymerase. The nuclear 665 target in signal transduction and its roles in brain ischemia-reperfusion injury. Molecular 666 Neurobiology 31:149-167. 
667 Strosznajder JB, Czapski GA, Adamczyk A, Strosznajder RP. 2012. Poly(ADP-ribose) 668 polymerase-1 in amyloid beta toxicity and Alzheimer's disease. Molecular Neurobiology 46:7866984.

670 Su YJ, Chang YW, Lin WH, Liang CL, Lee JL. 2015. An aberrant nuclear localization of E671 cadherin is a potent inhibitor of Wnt/ $\beta$-catenin-elicited promotion of the cancer stem cell 672 phenotype. Oncogenesis 4(6):e157. doi: 10.1038/oncsis.2015.17

673 Thomassin H, Jacobson MK, Guay J, Verreault A, Aboul-ela N, Menard L, Poirier GG. 1990. An 674 affinity matrix for the purification of poly(ADP-ribose) glycohydrolase. Nucleic Acids Research 675 18(16):4691-4694.

676 Thomassin H, Ménard L, Hengartner C, Kirkland JB, Poirier GG. 1992. Poly(ADP-ribosyl)ation 677 of chromatin in an in-vitro poly(ADP-ribose)-turnover system. Biochimica et Biophysica Acta 678 1137:171-181.

679 Tricaud N, Perrin-Tricaud C, Brusés JL, Rutishauser U. 2005. Adherens junctions in myelinating 680 Schwann cells stabilize Schmidt-Lanterman incisures via recruitment of p120 catenin to E681 cadherin. The Journal of Neuroscience 25:3259-3269.

682 Valentijn LJ, Baas F, Wolterman RA, Hoogendijk JE, van den Bosch NH, Zorn I, Gabreëls-Festen 683 AW, de Viser M, Bolhuis PA. 1992. Identical point mutations of PMP-22 in Trembler-J mouse 684 and Charcot-Marie-Tooth disease type 1A. Nature Genetics 2(4):288-291.

685 Virag L, Szabo C. 2002. The therapeutic potential of poly(ADP-ribose) polymerase inhibitors. 686 Pharmacological Reviews 54(3)375-429.

687 Vis JC, Schipper E, de Boer-van Huizen RT, Verbeek MM, de Waal RM, Wesseling P, Ten 688 Donkelaar HJ, Kremer B. 2005. Expression pattern of apoptosis-related markers in Huntington's 689 disease. Acta Neuropathologica 109:321-328. doi: 10.1007/s00401-004-0957-5

690 Vyas S, Chesarone-Cataldo M, Todorova T, Huang YH, Chang P. 2013. A systematic analysis of 691 the PARP protein family identifies new functions critical for cell physiology. Nature 692 Communications 4:2240. doi: 10.1038/ncomms3240 
693 Vyas S, Matic I, Uchima L, Rood J, Zaja R, Hay RT, Ahel L, Chang P. 2005. Family-wide 694 analysis of poly(ADP-ribose) polymerase activity. Nature Communications 5:4426. doi: $69510.1038 /$ ncomms5426

696 Wilson K, Walker J. 2005. Principles and techniques of Biochemistry and Molecular Biology. 6th 697 Ed. Cambridge University Press.

698 Ye F, Chen Y, Hoang T, Montgomery RL, Zhao XH, Bu H, Hu T, Taketo MM, van Es JH, Clevers 699 H, Hsieh J, Bassel-Duby R, Olson EN, Lu QR. 2009. HDAC1 and HDAC2 regulate 700 oligodendrocyte differentiation by disrupting the $\beta$-catenin-TCF interaction. Nature Neuroscience 701 12(7):829-838.

702 Yeh TY, Meyer TN, Schwesinger C, Tsun ZY, Lee RM, Chi NW. 2006. Tankyrase recruitment to 703 the lateral membrane in polarized epithelial cells: regulation by cell-cell contact and poly(ADP704 ribosyl)ation. Biochemical Journal 399:415-425.

705 Wang X, Sekine Y, Byrne AB, Cafferty WBJ, Hammarlund M, Strittmatter M. 2016. Inhibition of 706 poly-ADP-ribosylation fails to increase axonal regeneration or improve functional recovery after 707 adult mammalian CNS injury. eNeuro 3(6):e0270-16.2016, 1-10. doi: 10.1523/ENEURO.0270$708 \quad 16.2016$

709 Zuchero JB, Fu MM, Sloan SA, Ibrahim A, Olson A, Zaremba A, Dugas JC, Wienbar S, 710 Caprariello AV, Kantor C, Leonoudakis D, Lariosa-Willingham K, Kronenberg G, Gertz K, 711 Soderling SH, Miller RH, Barres BA. 2015. CNS myelin wrapping is driven by actin 712 disassembly. Developmental Cell 34(2):152-167. 

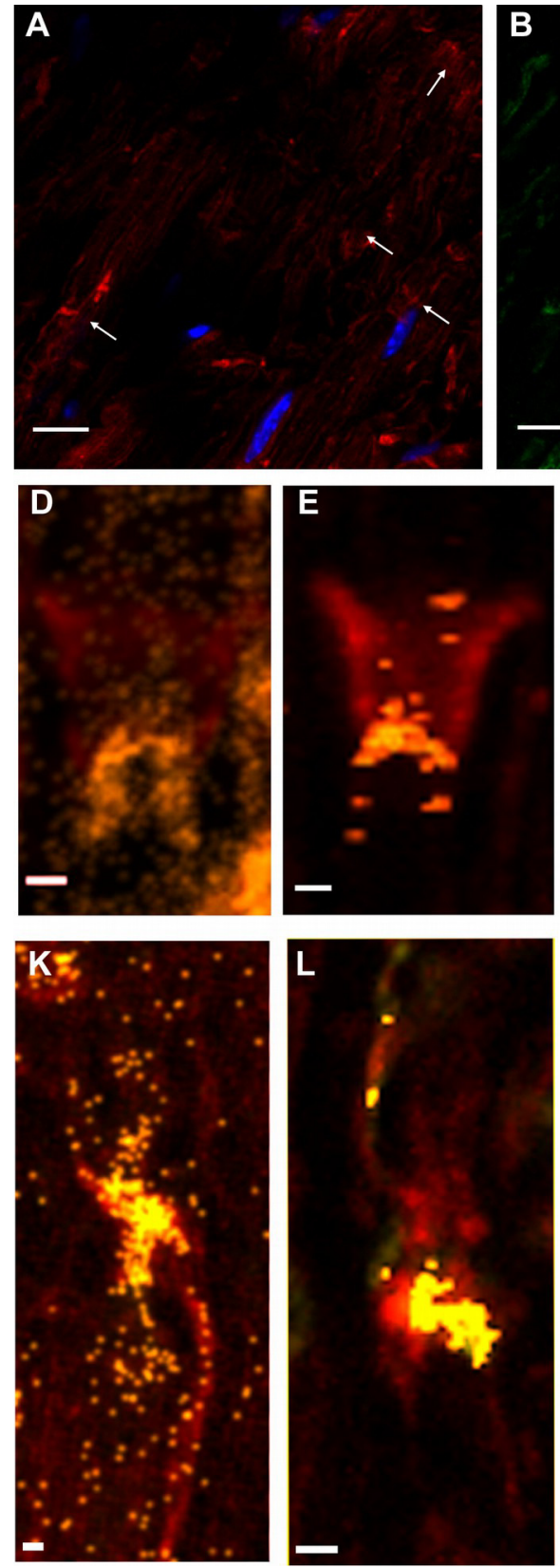

B
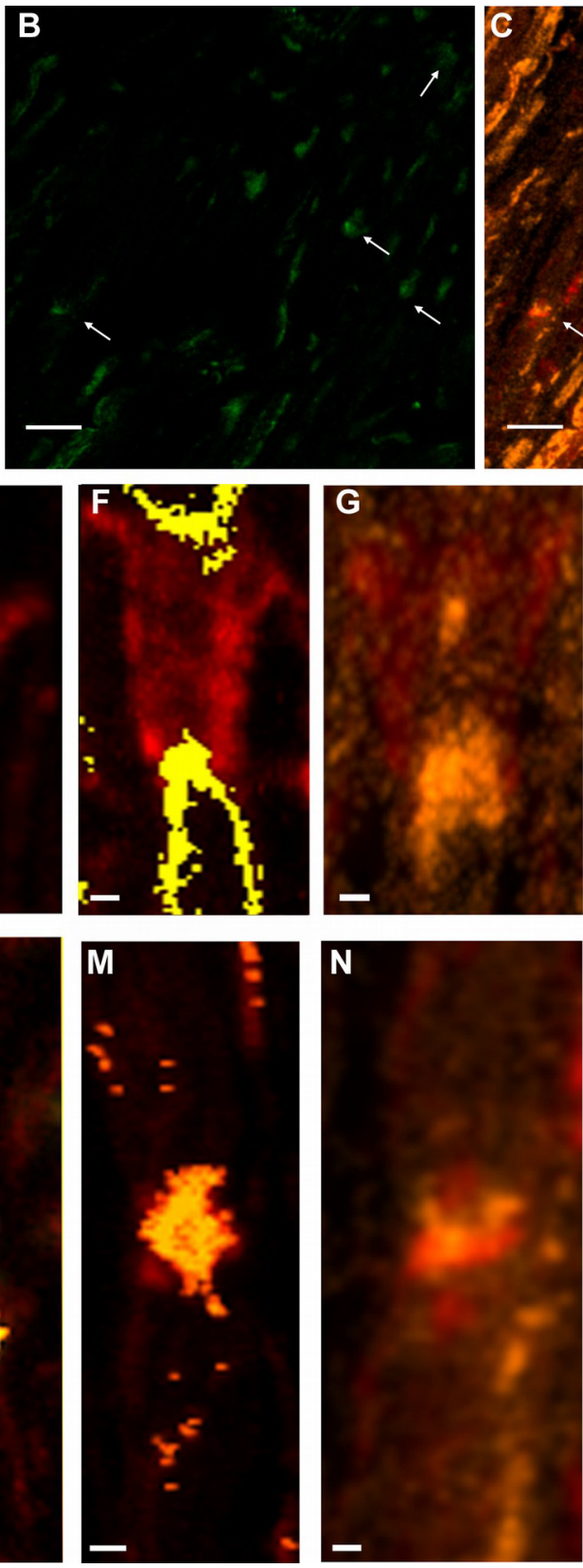
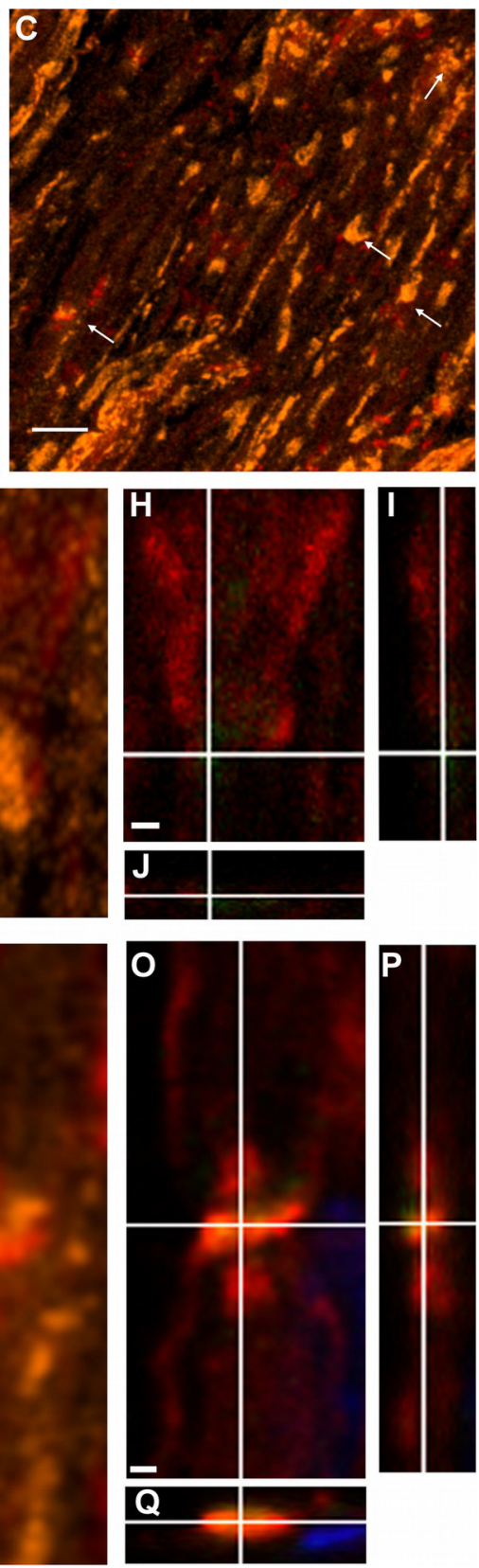

Figure 1. (See the legend on next page). 
714 Figure 1. Poly-ADP-ribose (PAR) was present in WT sciatic nerves, 715 particularly in non-compact myelin regions. A conserved color code has been 716 used in all the Figures. Green: PAR, red: F-actin; blue: DAPI; yellow/orange: F717 actin-PAR colocalization highlight. All the images were obtained using BD anti718 PAR antibody. A-C: 100x sciatic nerve overview. A,B: 100x single confocal 719 slices; C: correspondent F-actin-PAR colocalization highlight 3D projection. 720 Bar: $10 \mu \mathrm{m}$. The arrows point to colocalization regions outlined in a single-plane 721 which are better interpreted in the context of the 3D projection. Channel 722 intensities could be enhanced to facilitate eye detection, but then the photographs 723 would not be comparable to those in Figure 3. D-G: 3-D projections of Schmidt724 Lanterman incisures extracted from $\mathbf{C}(\mathbf{D}, \mathbf{G})$ and from other stacks. H-J: XY, XZ 725 and $Y Z$ cuts of the Schmidt-Lanterman incisures observed in G. K-N: 3-D 726 projections of paranode regions extracted from $\mathbf{C}(\mathbf{N})$ and from other stacks. O727 Q: $X Y, X Z$ and $Y Z$ cuts of the paranodes observed in $\mathbf{N}$. Bar: $1 \mu \mathrm{m}$. This result 728 was observed in 7 independent experiments. 


\section{PeerJ}


Figure 2.(See the legend on next page) 
730

731

732

733

734

735

736

737

738

739

740

741

742

743

744

745

746

747

748

749

750

751

752

753

754

755

756

757

Figure 2. F-actin increase inTr-J mice sciatic nerves was paralleled by PAR increase. The color code is maintained. Green: PAR, red: F-actin, blue: DAPI; yellow: colocalization highlighter mask. A-C: WT nerve; D-F: Tr-J nerve. 3-D reconstructions from 100x confocal stacks. Bar. $15 \mu \mathrm{m}$. GI: relative quantitation of cytoplasmic F-actin and PAR from 3 independent experiments with paired animals (WT and Tr-J siblings). All PAR was detected with BD anti-PAR antibody. Confocal microscopy images were taken under identical conditions for WT and Tr-J in each experiment, using as a reference the control without primary antibody. F-actin (red) and PAR (green) signal strength were measured along the fiber diameter in DAPInegative ROls. The length of the ROI was normalized and divided into 10 equal parts. It was assumed that the intervals from 0 to $30 \%$ and from 70 to $100 \%$ correspond to Schwann cells (SC) whereas the 30 to $70 \%$ range corresponds to axons. G: Relative intensity of F-actin and PAR signals along the normalized fiber diameter. Data were averaged by confocal stack $(n=12$ WT and $n=12$ Tr-J stacks from three independent experiments). Then they were normalized by mean WT axon F-actin and mean WT axon PAR of the corresponding experiment and expressed as stack mean \pm s.e.m.. H: Relative intensity of F-actin and PAR in the axons of WT (13132 measurements) and Tr-J (9409 measurements) mice; $n=12$ stacks. Mean \pm s.e.m. I: Counterpart of $\mathbf{H}$ in Schwann cells (WT: 8483 measurements and TrJ: 5983 measurements; $n=12$ stacks). J: Relative intensity of nuclear PAR in WT and Tr-J slices ( $n=55$ and 50 slices from 10 and 11 stacks respectively, from 3 independent experiments). Mean \pm s.e.m. (G-J): Data were normalized by WT axonal actin and WT axonal PAR (G-I) or WT nuclear PAR intensity $(\mathbf{J}) .\left(^{* * *}\right): P<0.001$ (Student's $t$ test). PAR increase was qualitatively observed in a total of 7 experiments. 



Figure 3. Even in still identifiable non-compact myelin regions of $\mathrm{Tr}-\mathrm{J}$ sciatic nerves, the distribution of PAR was altered. The color code is maintained. Green: PAR, red: F-actin, blue: DAPI; yellow: colocalization highlighter mask. All the images were obtained using BD anti-PAR antibody. A-C. 100x Tr-J sciatic nerve overview [comparable to WT sciatic nerve from Figure 1A-C]. A,B: 100x single confocal slices; C: corresponding F-actin-PAR colocalization highlight 3D projection. Bar: $10 \mu \mathrm{m}$. D-I

764 and J-O: representative paranode-like and Schmidt-Lanterman-like structures 765 extracted from C. D-F: $X Y, X Z$ and $Y Z$ cuts of a paranode. G,H: single slices at 766 different z-positions showing F- actin-PAR colocalization. I: 3-D reconstruction of the 767 paranode region. J-L: $X Y, X Z$ and $Y Z$ cuts of a Schmidt-Lanterman. $\mathbf{M}, \mathbf{N}$ : single slices 768 at different z-positions showing F-actin-PAR colocalization. O: 3-D reconstruction of 769 the paranode region. Bar: $1 \mu \mathrm{m}$. Altered PAR distribution was qualitatively observed 770 in a total of 7 experiments. 